This item was submitted to Loughborough's Research Repository by the author.

Items in Figshare are protected by copyright, with all rights reserved, unless otherwise indicated.

\title{
A method for quantification of the effects of size and geometry on the microstructure of miniature interconnects
}

PLEASE CITE THE PUBLISHED VERSION

https://doi.org/10.1007/s11664-013-2907-2

\section{PUBLISHER}

Springer $@$ TMS

VERSION

AM (Accepted Manuscript)

\section{PUBLISHER STATEMENT}

This work is made available according to the conditions of the Creative Commons Attribution-NonCommercialNoDerivatives 4.0 International (CC BY-NC-ND 4.0) licence. Full details of this licence are available at: https://creativecommons.org/licenses/by-nc-nd/4.0/

\section{LICENCE}

CC BY-NC-ND 4.0

\section{REPOSITORY RECORD}

Xiong, Hua, Zhiheng Huang, and Paul P. Conway. 2019. "A Method for Quantification of the Effects of Size and Geometry on the Microstructure of Miniature Interconnects". figshare. https://hdl.handle.net/2134/26831. 


\title{
A Method for Quantification of Microstructure in Miniaturized Interconnects with Size and Geometry Effects
}

\author{
Hua Xiong ${ }^{1}$, Zhiheng Huang ${ }^{1 *}$, Paul Conway ${ }^{2}$, Jim Robinson $^{3}$ \\ ${ }^{1}$ School of Physics and Engineering, Sun Yat-sen University, \\ 135 West Xingang Road, Guangzhou, China \\ ${ }^{2}$ Wolfson School of Mechanical and Manufacturing Engineering, Loughborough University, \\ Loughborough, Leicestershire, LE11 3TU, United Kingdom \\ ${ }^{3}$ National Physical Laboratory, Teddington, TW11 0LW, United Kingdom \\ *Tel./Fax: +86 (0)20 84110530 \\ E-mail: hzh29@mail.sysu.edu.cn
}




\section{ABSTRACT}

The heterogeneity of microstructure has significant impacts on the material properties of ultrafine interconnects, and thus should be quantified and used to facilitate high-fidelity reliability predictions. In order to address this challenge, a method based on autocorrelation and singular value decomposition for quantitative microstructural characterization is proposed in this work. The method is applied to study the size and geometry effects on the microstructure and the stress state in ultrafine $\mathrm{Sn} 37 \mathrm{~Pb}$ solder joints. The degree of the microstructural heterogeneity related to the preferred growth directions of the phases is quantified with a scalar microstructural index by using this method. It is found that the degree of microstructural heterogeneity increases with the decrease of the standoff height, and is higher in the hourglass-shaped solder joints. The maximum von Mises stress is lower in the hourglass-shaped joints in most cases studied in this work, which indicates a higher strength and longer lifetime than the barrel-shaped joints. The average von Mises stress increases almost monotonically with the degree of the microstructural heterogeneity. The strong correlation between the microstructural index and the average von Misse stress is confirmed by a nonlinear regression analysis using artificial neural network. Based on the microstructural index, the mechanical behavior of the ultrafine interconnects can be predicted more accurately.

Keywords: Ultrafine interconnects, microstructure, size and geometry effects, reliability, microstructure-property relationship. 


\section{INTRODUCTION}

Highly miniaturized interconnects are critical to enable co-design and comanufacturing of devices and their packages for the next generation packaging technologies, such as 3D packaging and system integration. However, as the dimensions of the interconnects continuously shrink from mesoscale down to nanoscale, the number of grains is obviously reduced and the sizes of the constituent phases are comparable to those of the interconnects. As a result, the material properties of the interconnects become more anisotropic and heterogeneous. Conventional lifetime prediction methods for materials with homogeneous properties, as widely used today, are no longer applicable to such ultrafine interconnects with highly heterogeneous microstructures.

Furthermore, new reliability issues can arise due to the substantial effects arising from the size and geometry of ultrafine interconnects on microstructural evolution [16]. For example, a significant increase in resistivity, i.e. by $29 \%$, was observed by Khoo et al. when the width of $\mathrm{Cu}$ line interconnects was reduced from $80 \mathrm{~nm}$ to 50 $\mathrm{nm}$ [2]. The higher resistivity was ascribed to the smaller grain sizes whose growth was restricted by the sidewall. The size and geometry effects on microstructure were found to be even more complicated in solder joints [4-6], since solders commonly possess multiple phases. In addition, the geometry of solder joints has revealed obvious impacts on reliability at a comparatively larger scale. Liu et al. found that hourglass-shaped $\mathrm{Sn} 37 \mathrm{~Pb}$ solder joints of $1.25 \mathrm{~mm}$ pad size improved fatigue lifetime by about $60 \%$ over the conventional barrel-shaped joints of the same pad size [7]. The question as to whether this effect will still be significant for joint size below a few tens of microns is of particular interest. To date, a complete understanding on the size and geometry effects is still lacking. In order to achieve high-fidelity reliability 
predictions for ultrafine interconnects, a novel microstructure-property relationship incorporating the size and geometry effects should be established. The first step towards this goal is to quantify the microstructure.

In the existing literature, local features of the microstructure of $\mathrm{Cu}$ or $\mathrm{Pb}$-free solders have been used for quantitative microstructural characterization [1-3, 8-11]. For example, Sidhu [9] and Dutta et al. [10] studied the effect of the interparticle spacing and the particle size of $\mathrm{Ag}_{3} \mathrm{Sn}$ particle on the creep behavior of $\mathrm{SnAg}$ and $\mathrm{SnAgCu}$ solders. A modified expression of a threshold stress incorporating the microstructural effect was obtained in these cases. However, their method for microstructural quantification is only applicable to the microstructures with uniform size and shape distributions of the constituent phases. Another method based on topological analysis was proposed by Gameiro et al. [12]. This method can quantify the connectivity of the complicated microstructures formed in eutectic $\mathrm{SnPb}$ or $\mathrm{SnBi}$ solders, but is still unable to describe the spatial distribution of the phases. As the microstructures in ultrafine interconnects are highly heterogeneous, local features and topological information for the microstructure are no longer adequate for high-fidelity reliability predictions. A better approach to quantify the heterogeneity of microstructure is required.

To address this challenge, a method based on autocorrelation [13] and Singular Value Decomposition (SVD) [14] is proposed in the present work. This method is generally applicable to a wide range of microstructures in electronic interconnects, e.g. the distributed particles of Intermetallic Compounds (IMCs) in Sn-based solder joints and the grain structures in $\mathrm{Cu}$ Through Silicon Vias (TSVs). In addition, it can work for the microstructures generated by both modeling and experimental work. To demonstrate the capability of this method, an example to quantify the microstructural 
heterogeneity in ultrafine $\mathrm{Sn} 37 \mathrm{~Pb}$ solder joints is provided. The structure of the paper is organized as follows. First, a model for simulating phase separation and coarsening in solder interconnects under shear stress is introduced. Secondly, a qualitative description of the size and geometry effects on the simulated microstructure and stress is given for a comparison with the quantitative results presented later. Thirdly, a method capable of quantifying the microstructural heterogeneity is described in detail. Finally, the method is applied to quantitatively study the nonlinear relationships among the size, geometry, microstructure and mechanical behavior of the miniaturized interconnects.

\section{MODELING METHODOLOGY}

As a detailed example to show the general applicability of our method, the phase field model developed by Dreyer and Müller [15] is employed in this work to simulate the phase separation and coarsening processes in ultrafine $\mathrm{Sn} 37 \mathrm{~Pb}$ solder joints. This model incorporates the strain energy as a driving force for microstructural evolution, and thus can predict the microstructure in solder joints under shear load when the joints are in service. The distribution of the mass fraction of $\mathrm{Sn}$, denoted as $C_{S n}$, is used as an order parameter to represent the morphology of the Sn-rich and $\mathrm{Pb}$-rich phases. The evolution of the morphology is obtained by solving the following governing equation with the finite element method [15]:

$$
\frac{\partial C_{S n}}{\partial t}+\frac{\partial}{\partial x_{i}}\left[M_{i j} \frac{\partial}{\partial_{j}}\left(\frac{\partial \psi}{\partial C_{S n}}-a_{k l} \frac{\partial^{2} C_{S n}}{\partial x_{k} \partial x_{l}}+\frac{\partial W_{s}}{\partial C_{S n}}\right)\right]=0
$$

where $M_{i j}$ is the mobility matrix, $a_{k l}$ is a matrix of the coefficients related to surface energy, $W_{s}$ is the strain energy density, and $\psi$ is the chemical free energy density obtained by using the thermodynamic calculation software package Thermo-Calc [16] 
with the solder database developed at NIST [17]. It should be noted that the Einstein summation convention is used throughout this paper.

The solder is modelled as a linear elastic material in the present work. The stress-strain constitutive equation is as follows:

$$
\sigma_{i j}=E_{i j k l} \varepsilon_{k l}^{e l}
$$

where $\sigma_{i j}$ is the stress tensor, $E_{i j k l}$ is the stiffness tensor, and $\varepsilon_{k l}^{e l}$ is the elastic strain tensor. The stress reaches equilibrium much faster than the evolution of microstructure, and thus the following static equation is used to solve for stress at every time step of the simulations:

$$
\frac{\partial \sigma_{i j}}{\partial x_{i}}=0
$$

The governing equations of the phase field model and the mechanical model are solved in $2 \mathrm{D}$ for ultrafine $\mathrm{Sn} 37 \mathrm{~Pb}$ solder joints. Two geometries of solder joints are studied in this work, i.e. the barrel shape and the hourglass shape, as illustrated in Fig.1. The left and right boundaries of the solder joints are assumed to be circular arcs, which are approximations of the joint surfaces determined by surface energy and gravitational energy. A shape factor $\kappa$ is introduced to quantitatively describe different geometries [18]:

$$
\kappa=\frac{S_{0}}{h d}-1
$$

where $S_{0}$ is the area of the cross section of the joints, $h$ is the standoff height, and $d$ is the diameter of the pad. The shape factor $\kappa$ can be regarded as a "dimensionless curvature", which can describe the geometrical characteristics independent of the joint size. $\kappa>0$ is for the barrel-shaped joints, and $\kappa<0$ is for the hourglass-shaped joints, as indicated in Fig. 1. The standoff height $h$ and the pad size $d$ are set to be the same, 
i.e. $h=d$. Eleven standoff heights ranging from $1.50 \mu \mathrm{m}$ to $8.00 \mu \mathrm{m}$ are studied for each geometry.

For the phase field equation, i.e. Eq. 1, a slight fluctuation of $C_{S n}$ around $63 \mathrm{wt} . \%$ is set as an initial condition to simulate the effect of temperature fluctuations on microstructural evolution. Six different initial conditions are studied for each configuration of size and geometry, i.e. the fluctuation of $C_{S n}$ at six different locations in the joints as indicated by the dots in Fig. 1. For the mechanical equations, i.e. Eqs. 2 and 3, the joints are assumed to have no initial stress. The boundary conditions are set as follows. For Eq. 1, a zero flux boundary condition is applied to all boundaries of the joints; for Eqs. 2 and 3, the bottom boundary is fixed while the left and right boundaries can deform freely, and a shear displacement is applied to the top boundary as illustrated in Fig. 1. The shear loads imposed on the solder joints in real electronic packages may vary with the joint size and geometry, even when they are placed at the same location. Therefore, the shear displacements of the solder joints close to real scenarios need to be obtained. For this purpose the thermal expansion in two packaging structures as shown in Fig. 2 is simulated. In this case the microstructures in the solder joints are not considered. The joints labelled with the same number in Fig. 2 are set to locate at the same position in the package. The simulation result is present in Fig. 3. For the joints at the same location, it is found that the shear displacements of the top boundary of the joints are only slightly influenced by the standoff height and geometry of the joints. In addition, the magnitude of the majority of the shear displacements predicted in this model is found to be around $0.01 \mu \mathrm{m}$. According to this result, a shear displacement of $0.01 \mu \mathrm{m}$ is applied to the top boundary of the solder joints in the coupled microstructure and stress simulations in the present work. 


\section{RESULTS AND DISCUSSIONS}

\section{A Qualitative Description of Size and Geometry Effects}

Fig. 4 demonstrates the simulated microstructures formed at $80 \mathrm{~s}$ during phase separation and coarsening at $125^{\circ} \mathrm{C}$ in $\mathrm{Sn} 37 \mathrm{~Pb}$ ultrafine interconnects with different sizes and geometries. The Sn-rich phase is shown in bright color, while the Pb-rich phase is in dark color. Both of the phases are found to grow preferentially along the boundaries of the joints. Such a morphology is comparable to the heterogeneous coarsening bands observed by Woodmansee et al. in a shear test of $\mathrm{Sn} 40 \mathrm{~Pb}$ specimens [19]. The phase growth in preferred directions is due to the combined effect from the shear stress and the constraints on diffusion imposed by the geometry of the joint surface. Therefore, the degree of microstructural heterogeneity will change when the size or geometry of the solder joints changes, which influences the stress state in the joints and the ratio of joint surface area to volume.

Referring to Fig. 4, the size and geometry effects on the microstructure can be described qualitatively. The phases present in smaller joints have a relatively larger size compared to the sizes of the joints. The increase of the relative size of the phases can result in a more heterogeneous phase distribution. For example, the middle region of the hourglass-shaped joint of $4.5 \mu \mathrm{m}$ standoff height can provide enough space for the phases to grow in more than one direction, as shown in Fig. 4g. In comparison, a (Pb-rich)-(Sn-rich)-(Pb-rich) sandwich structure is formed in the middle region of the joint when the standoff height shrink to $1.5 \mu \mathrm{m}$, as shown in Fig. 4e. In addition to the size effect, the hourglass-shaped geometry can also enhance the phase growth in the preferred directions. The phases at the centre of the barrel-shaped joints seem to grow in comparatively random directions, as shown in the square region marked in

Fig. 4d. In contrast, the phases tend to grow along the horizontal and vertical 
directions at the centre of the hourglass-shaped joints, as indicated by the square region marked in Fig. 4h. However, the information provided by this qualitative description is limited and cannot be used to compare the degree of heterogeneity among the simulated microstructures. In order to circumvent this dilemma, a method capable of quantifying the microstructural heterogeneity will be introduced in the following section.

Fig. 5 demonstrates the distributions of von Mises stress in the solder interconnects with the microstructures shown in Fig. 4. The maximum and average von Mises stresses are shown overlaid the specific joint. For a comparison, the stress distributions in the joints without microstructure are also given as shown in Fig. 6. Local stress concentration in the Sn-rich phase can be clearly observed by comparing the stress distributions in Fig. 5e with the corresponding microstructure in Fig. 4e. Figs. 5 and 6 show that the region with high von Mises stresses, e.g. over $40 \mathrm{MPa}$, will become scattered and result in a decrease of the average stress, if the microstructure is taken into consideration. In addition, by considering microstructure, the magnitude of the maximum von Mises stress is also obviously changed. For example, the maximum stress can be increased by over $130 \%$, by comparing Fig. 5c to $6 \mathrm{c}$, or be reduced by $22 \%$, by comparing Fig. $5 \mathrm{a}$ to $6 \mathrm{a}$. Moreover, the locations of maximum stress in the hourglass-shaped joints, as indicated by the arrows illustrated in Figs. 5f-h and 6f-h, are shifted from the boundaries into the interior of the joints when microstructure is considered. These observations indicate that both the magnitude and the spatial distribution of the von Mises stress are highly dependent on the microstructures formed in the joints. Therefore, the aforementioned size and geometry effects on the microstructure can influence the mechanical behavior of the joints indirectly. It is the objective of next section to examine whether the 
microstructural effect on the mechanical behavior of the interconnects can be described by a quantified parameter representing the microstructural heterogeneity.

\section{A Quantitative Analysis of Size and Geometry Effects}

The aforementioned size and geometry effects are quantitatively investigated in this section. First, a method for the quantification of microstructure that combines techniques for microstructural feature extraction and data dimension reduction is described in detail. A nonlinear regression method based on Artificial Neural Network (ANN) is also be introduced. Then the validity of the microstructural quantification method is discussed by comparing the quantified results with the microstructures shown in Fig. 4. Finally, a relationship among size, geometry, microstructure and mechanical behaviours of miniaturized interconnects is developed and analysed by using ANN.

\section{$\underline{\text { A Microstructural Quantification Method }}$}

The extraction of microstructural features, e.g. grain orientation distribution [20], and volume fraction, size, preferred growth direction of a specific phase, is conducted by calculating the autocorrelation matrix, which is a widely used statistical description of microstructures [13, 21, 22].

The microstructural image of $m$-by- $n$ pixels, either from modeling or experimental work, is represented by an $m$-by- $n$ matrix $C$. Each component of the matrix $C$ is the value of a local feature of the microstructure at the specific location. A representative microstructure studied in this work and the corresponding pixels are schematically illustrated in Fig. 7 as an example. The local feature is chosen to be the mass fraction of $\mathrm{Sn}$, and the spatial interval of the pixels is set to be $0.01 \mu \mathrm{m}$ in both the horizontal and the vertical directions. Then the component $M_{i j}$ of the autocorrelation matrix is calculated as follows: 


$$
M_{i j}=\sum_{k}^{m} \sum_{l}^{n} C_{k l} C_{(k+i-r)(l+j-s)} / \sum_{k}^{m} \sum_{l}^{n} N_{k l} N_{(k+i-r)(l+j-s)}
$$

where $r=[(m-1) / 2]$ and $s=[(n-1) / 2]$ are the largest integers no greater than $(m-1) / 2$ and $(n-1) / 2$ respectively, $M_{r s}$ is the component at the centre of the autocorrelation matrix; $i \in[1,2 r+1]$ and $j \in[1,2 s+1]$ are integers; $N_{k l}$ is 1 within the domain of microstructure, e.g. the barrel-shaped domain shown in Fig. 7, and is otherwise 0 . The component $M_{i j}$, represents the probability that the same microstructural feature, e.g. the Sn-rich phase, appears at both the start and the end points of a vector $(i-r, j-s)$, which is randomly placed in the microstructural image. A schematic plot of the vector $(i-r, j-s)$ is illustrated in Fig. 7.

The autocorrelation matrix commonly contains thousands of variables, i.e. the components of the matrix. Most of these variables are interdependent [22]. As such, it is difficult and time consuming to fit a relationship among microstructure, size, geometry, and mechanical behavior, if the autocorrelation matrix is directly used to represent the microstructure. Principal Component Analysis (PCA) based on SVD [14] is utilized to extract the most essential variables that determine the main characteristics of the microstructure and reduce the number of variables. The autocorrelation matrices of the simulated microstructures for different sizes and geometries are first averaged to obtain a benchmark $A$ for the quantification of these microstructures. SVD is then conducted on the benchmark [14]:

$$
A=U S V^{T}
$$

where the columns of matrices $U$ and $V$ are orthogonal bases of the variable space, and the components of the diagonal matrix $S$ are the singular values that represent the importance of the corresponding bases in describing the microstructure. The singular 
values are ranked in descending order and the cumulative contribution rate of the first $p$ sets of singular value and bases is defined as:

$$
Q^{p}=\sum_{i}^{p} S_{i i} / \sum_{i}^{m} S_{i i}
$$

If $Q^{p}$ is large, e.g. over $90 \%$, then the first $p$ singular values and their corresponding bases can describe the original microstructure with negligible loss of information. Finally, the autocorrelation matrix $M$ is projected onto the orthogonal bases to obtain a matrix $P$ :

$$
P=U^{T} M V
$$

The matrix composed of the first $p \times p$ components of $P$, denoted as $\hat{P}$, is used to calculate a scalar microstructural index $\delta$ by using the Frobenius norm [14] as follows:

$$
\delta=|\hat{P}|_{F}=\sqrt{\sum_{i}^{p} \sum_{j}^{p} P_{i j}^{2}}
$$

The value of $\delta$ will decrease to 0 when the microstructural characteristics represented by $M$ are infinitely close to those represented by the benchmark $A$, and will increase otherwise. For example, if the benchmark represents a microstructure with the phase growth in random directions, then a microstructure with the phase growth in preferred directions will correspond to a high value of $\delta$.

\section{$\underline{\text { A Nonlinear Regression Method }}$}

The nonlinear relationships among the microstructural index $\delta$, the standoff height $h$, the shape factor $\kappa$ given by Eq. 4 and the average stress $\sigma^{a v e}$ are fitted and analyzed by using ANN [23]. The output variable $v^{\text {out }}$, i.e. the microstructural index $\delta$ or the average stress $\sigma^{a v e}$, calculated by ANN is expressed as follows [23]:

$$
v^{\text {out }}=\sum_{i}^{S} w_{i}^{\text {out }} u_{i}+b^{\text {out }}
$$


where $u_{i}$ is the output of the $i$ th neuron of the ANN given by:

$$
u_{i}=\tanh \left(\sum_{j}^{R} w_{i j}^{i n} v_{j}^{i n}+b_{i}^{i n}\right)
$$

In Eqs. 10 and $11, v_{j}^{\text {in }}$ are the input variables, i.e. the standoff height $h$ and the shape factor $\kappa$ when $v^{\text {out }}=\delta$, or the microstructural index $\delta$ when $v^{\text {out }}=\sigma^{\text {ave }} ; R$ and $S$ are the numbers of the input variables and the neurons respectively; and $w_{i}^{\text {out }}, b^{\text {out }}$, $w_{i j}^{i n}, b_{i}^{i n}$ are the parameters that need to be adjusted to minimize the error function as follows:

$$
e=\frac{1}{T} \sqrt{\sum_{i}^{T}\left(v_{i}^{\text {sim }}-v_{i}^{\text {out }}\right)^{2}}
$$

where $T$ is the number of the samples used to fit the relationship, $v_{i}^{\text {sim }}$ and $v_{i}^{\text {out }}$ are the output variables for the $i$ th sample obtained by simulation and predicted by ANN respectively. The prediction accuracy of the ANN can be examined by conducting linear regression analysis on $v_{i}^{\text {sim }}$ and $v_{i}^{\text {out }}$ for $i \in[1, T]$, i.e. all of the samples. The residual $\varepsilon_{i}$ defined as follows is used to evaluate the prediction error for the $i$ th sample.

$$
\varepsilon_{i}=v_{i}^{\text {sim }}-v_{i}^{\text {out }}
$$

\section{Quantification of Microstructural Heterogeneity}

Fig. 8a demonstrates the visualization of the benchmark $A$, which represents the microstructural characteristics shared in common by all of the microstructures studied in this work. This figure can be interpreted as follows. A vector pointing from coordinate $(0,0)$ to the coordinate of the component $A_{i j}$ should be drawn first, as shown in Fig. 8a. Then the value of $A_{i j}$ represents the probability of finding the Snrich phase simultaneously at the start and the end points of this vector when it is randomly placed in the microstructural image, e.g. as shown in Fig. 7. The major 
features of the microstructures can be retrieved from Fig. 8a. For example, the volume fraction of the Sn-rich phase can be represented by the autocorrelation value at the centre of Fig. 8a [21]. In addition, the average width of the strip-shaped Sn-rich phase can be estimated by the radius of the dark "ring" surrounding the centre of Fig. 8a, since the ring represents extremely low values of the autocorrelation. Furthermore, the length of the interface between the $\mathrm{Sn}$-rich and the $\mathrm{Pb}$-rich phases per unit area can be calculated by using the spatial derivative of the autocorrelation at the centre of Fig. 8a [22].

The underlying information of the microstructural characteristics, e.g. the phase distribution, can be further clarified by conducting SVD-based PCA on the benchmark $A$. The first three singular values obtained in this work are $S_{11}=57.5$, $S_{22}=1.5$, and $S_{33}=0.5$. Since $S_{11}$ is far greater than $S_{22}$ and $S_{33}$, the phase distribution in the ultrafine solder joints is mainly determined by the first principal component (PC1) of the benchmark $A$, which is calculated by $U_{i 1} S_{11} V_{j 1}^{T}$ and visualized in Fig. 8b. The cruciform pattern in Fig. $8 b$ indicates that the Sn-rich phase tends to grow in the directions parallel to the $X$ and the $Y$ axes. The preferred growth directions are in agreement with the qualitative description of Fig. 4. As such, the benchmark $A$ contains the major characteristics of the heterogeneous microstructures studied in this work. The degree of the microstructural heterogeneity for different joint sizes and geometries are then quantified by using the benchmark $A$ as follows. The first 17 singular values and their corresponding orthogonal bases of the benchmark $A$ are used to calculate the microstructural index $\delta$ given by Eq. 9 . In this case, a high cumulative contribution rate $Q^{p}$, i.e. over $99.99 \%$, can be obtained, and thus the loss of microstructural information is negligible.

$\underline{\text { Size and Geometry Effects on Microstructure }}$ 
Fig. 9 demonstrates the relationship between the microstructural index $\delta$ and the standoff height $h$, for the barrel-shaped and the hourglass-shaped solder joints respectively. It is clear that the hourglass-shaped solder joints have a higher value of $\delta$ than the barrel-shaped joints for all of the studied standoff heights. In order to examine that, a higher value of $\delta$ indicates a higher degree of the microstructural heterogeneity, and for comparison the PC1 images corresponding to the barrel-shaped and the hourglass-shaped joints shown in Fig. 4a and e are plotted in Fig. 8c and d respectively. The PC1 for the barrel-shaped joint, as shown in Fig. 8c, is similar to that of the benchmark as shown in Fig. 8b, except for four additional regions of high autocorrelation value. In contrast, the PC1 for the hourglass-shaped joint, as shown in Fig. 8d, is evidently different from Fig. 8 b. It is found that the bright regions, i.e. the regions of high autocorrelation value, concentrate near the centre, the top, and the bottom of this PC1 image. In addition, two "gaps" of low autocorrelation value, whose central lines locate at $Y= \pm 0.5 \mu \mathrm{m}$, are observed in Fig. 8d. The two gaps reflect a highly heterogeneous microstructure, i.e. the (Pb-rich)-(Sn-rich)-(Pb-rich) sandwich structure described in the above section, since the distance between the two layers of the Pb-rich phase is close to $0.5 \mu \mathrm{m}$ as indicated in Fig. 4e. Therefore, it is confirmed that the larger microstructural index $\delta$ of the hourglass-shaped joint indicates a more heterogeneous phase distribution. As such, Fig. 9 can be explained as follows: the degree of microstructural heterogeneity is found to increase with the decrease of the standoff height, and is higher in the hourglass-shaped than barrelshaped joints of the same standoff height.

The relationship among the size, geometry and microstructure of the solder joints is further analyzed by using ANN. The standoff height $h$ and the shape factor $\kappa$ are used to predict the microstructural index $\delta$. Fig. 10 demonstrates a comparison of the 
microstructural index predicted by $\mathrm{ANN}$, i.e. $\delta^{a n n}$, and calculated based on the simulation results, i.e. $\delta^{\text {cal }}$. It is clear that the linear regression line for the calculated and the predicted microstructural indices is close to the dashed line that represents the relationship of $\delta^{a n n}=\delta^{c a l}$. In addition, the linear correlation coefficient is close to 1 . Therefore, the microstructural index $\delta$ is strongly correlated to the size and geometry of ultrafine solder joints. However, the accuracy of the prediction apparently drops when the microstructural index $\delta$ is larger than 3, as shown in Fig. 10. Referring to Fig. 9, the data for $\delta \geq 3$ are all corresponding to the hourglass-shaped joints with standoff height below $2 \mu \mathrm{m}$. By comparing the data points to the curves fitted by ANN as shown in Fig. 9, it is found that the low prediction accuracy of these data is due to large variances of $\delta$. These variances are induced by slight differences in the initial distributions of the mass fraction of Sn, which simulates the effect of temperature fluctuations. Since the same initial conditions are imposed for all configurations of size and geometry, the magnitude of the variances of $\delta$ is determined by the size and geometry of the joints. This result indicates that it is more difficult to predict and thereby design the microstructure in ultrafine solder joints of the hourglass geometry.

\section{$\underline{\text { Size and Geometry Effects on Mechanical Behavior }}$}

Fig. 11 demonstrates the maximum and the average von Mises stress, i.e. $\sigma^{\max }$ and $\sigma^{a v e}$, in the solder joints with different standoff heights and geometries, respectively. The results for both the joints with and without microstructure are plotted for a comparison. Both $\sigma^{\max }$ and $\sigma^{a v e}$ are found to be higher in smaller joints when the microstructure is not considered. However, it is difficult to predict the maximum stress $\sigma^{\max }$ in the joints with microstructure, since $\sigma^{\max }$ is highly sensitive to 
minute microstructural changes. To be more specific, Fig. 11a shows that $\sigma^{\max }$ in the barrel-shaped joints keeps increasing when the standoff height $h$ decreases from $8 \mu \mathrm{m}$ to $1.85 \mu \mathrm{m}$, but $\sigma^{\max }$ suddenly drops when $h$ shrinks down to $1.5 \mu \mathrm{m}$ and $1.65 \mu \mathrm{m}$. In contrast, it is relatively easy to predict the microstructural effect on the average stress $\sigma^{a v e}$. Since the $\mathrm{Pb}$-rich phase has a lower Young's modulus, it deforms more easily than the Sn-rich phase. In this case, the shear displacement of the joints with microstructure is mainly attributed to the deformation of the $\mathrm{Pb}$-rich phase, while the deformation of the Sn-rich phase is mitigated. As a result, $\sigma^{\text {ave }}$ is generally reduced compared to the case without microstructure as shown in Fig. $11 \mathrm{~b}$. However, $\sigma^{\text {ave }}$ still increases with the decrease of the standoff height for the joints with microstructure.

In addition to the size effect, the geometry effect on the mechanical behavior is also significant. The barrel-shaped joints always have a much higher value of $\sigma^{\max }$, i.e. by more than $100 \mathrm{MPa}$, than the hourglass-shaped joints when the microstructure is not considered. Such comparatively high maximum stress for the barrel-shaped geometry is induced by the local stress concentration at one of the corners, as indicated by the arrows shown in Figs. 6a-d. Even when the microstructure is taken into account, $\sigma^{\max }$ still remains lower for the hourglass-shaped joints in most cases as shown in Fig. 11a. This could be a possible explanation for the higher strength and longer lifetime of the hourglass-shaped joints experimentally observed by Liu et al. [7]. In contrast, $\sigma^{a v e}$ is found to be higher in the hourglass-shaped joints whether the microstructure is considered or not. Moreover, the data points in Fig. 11b demonstrate larger variances of $\sigma^{\text {ave }}$ for the hourglass-shaped joints, especially for standoff heights below $2 \mu \mathrm{m}$. The variances of $\sigma^{\text {ave }}$ are induced by the variations of the microstructure as illustrated in Fig. 9. Therefore, the hourglass-shaped joints can also have a large variance in other material properties, such as resistivity and thermal 
conductivity. Such variation may cause reliability issues, especially in high density packages containing a large number of joints.

Considering Figs. 9, 11b and the above discussions, it is evident that the size and geometry effects on the microstructural index $\delta$ and the average von Mises stress $\sigma^{\text {ave }}$ are similar. However, whether $\delta$ can describe the microstructural effect on the mechanical behavior, or more specifically, whether the mechanical properties can be predicted accurately based on $\delta$, is still unknown and needs further examination. For this purpose the relationship between $\delta$ and $\sigma^{a v e}$ is investigated. The data points in Fig. 12a reveal a unified relationship between $\sigma^{a v e}$ and $\delta$ for both the barrel-shaped and the hourglass-shaped geometries. It should be noted that usually three variables, i.e. the standoff height $h$, the shape factor $\kappa$, and the microstructural index $\delta$, are needed to determine the stress state in the joints. However, it is already confirmed that $\delta$ has a strong correlation to $h$ and $\kappa$ as shown in Fig. 10. In this case the microstructural index $\delta$ contains the information for the size and geometry, and thus can determine the average stress $\sigma^{\text {ave }}$ as shown in Fig. 12a. This relationship is fitted by using ANN and illustrated as a solid-line curve. It is noted that a nearly monotonic relationship exists between $\sigma^{a v e}$ and $\delta$, which is beneficial for the inverse design of microstructure. Fig. $12 \mathrm{~b}$ illustrates the residuals of this ANN, i.e. the differences between the simulated average stress and those fitted, as a function of the standoff height $h$ for the barrelshaped and the hourglass-shaped joints respectively. It is found that the data points are randomly dispersed around the zero-residual line. The magnitude of the residuals is also independent of the size and geometry of the joints. This result confirms that the microstructure-induced variance of $\sigma^{\text {ave }}$ can be predicted by $\delta$. Accordingly, incorporating the microstructural index $\delta$ into prediction models such as ANN can be 
used to improve the prediction accuracy for the mechanical behavior of ultrafine interconnects.

\section{CONCLUSIONS}

A method for quantifying the microstructure in ultrafine interconnects is proposed and applied to study the size and geometry effects on the microstructure and the stress state in ultrafine $\mathrm{Sn} 37 \mathrm{~Pb}$ solder joints. The following conclusions can be drawn:

1. The degree of the microstructural heterogeneity, i.e. the growth of the phases in preferred directions, can be quantified with a scalar microstructural index by using the proposed method;

2. The degree of microstructural heterogeneity is found to increase with the decrease of the standoff height. For the same standoff height, the hourglass-shaped joints have more heterogeneous microstructures than the barrel-shaped joints;

3. The hourglass-shaped joints have a lower maximum von Mises stress in most cases studied in this work, and thus could possibly possess a higher strength and longer lifetime than the barrel-shaped counterpart. The average von Mises increases nearly monotonically with the degree of the microstructural heterogeneity;

4. The strong correlation between the microstructural index and the average von Mises stress is confirmed by the nonlinear regression analysis using ANN. Based on the microstructural index, the mechanical behavior of the ultrafine interconnects can be predicted more accurately. 


\section{ACKNOWLEDGEMENTS}

The authors wish to acknowledge the financial supports from the National Natural Science Foundation of China (NSFC) under grant no. 51004118, the Pearl River New Science Star Program of Guangzhou under grant no. 2012J2200074, the Fundamental Research Funds for the Central Universities from the Ministry of Education (SYSU internal grant no. 30000-3161451), the Scientific Research Foundation for the Returned Overseas Chinese Scholars, State Education Ministry (SYSU internal grant no. 30000-4105346).

\section{REFERENCES}

1. D. Kwon, H. Park, and C. Lee, Thin Solid Films 475, 58 (2005).

2. K. Khoo, J. Onuki, T. Nagono, S. Tashiro, Y. Chonan, H. Akahoshi, T. Haba, T. Tobita, M. Chiba, and K. Ishikawa, Mater. Trans. 48, 2703 (2007).

3. D. Josell, S.H. Brongersma, and Z. Tokei, Annu. Rev. Mater. Res. 39, 231 (2009).

4. Z. Huang, P.P. Conway, E. Jung, R.C. Thomson, C. Liu, T. Loeher, and M. Minkus, J. Electron. Mater. 35, 1761 (2006).

5. B.S.S.C. Rao, D.M. Fernandez, V. Kripesh, and K.Y. Zeng, 12th Electronics Packaging Technology Conference (Singapore; IEEE 2010), pp. 423.

6. C. Chen, L. Zhang, J. Zhao, L. Cao, and J.K. Shang, J. Electron. Mater. 41, 2487 (2012).

7. X. Liu, and G. Lu, IEEE Trans. Compon. Packag. Technol. 26, 455 (2003).

8. R.S. Sidhu, and N. Chawla, Metall. Mater. Trans. A 39A, 340 (2008).

9. R.S. Sidhu, X. Deng, and N. Chawla, Metall. Mater. Trans. A 39A, 349 (2008).

10. I. Dutta, D. Pan, R.A. Marks, and S.G. Jadhav, Mater. Sci. Eng. A 410-411, 48 (2005).

11. M. Maleki, J. Cugnoni, and J. Botsis, Acta Mater. 61, 103 (2013). 
12. M. Gameiro, K. Mischaikow, and T. Wanner, Acta Mater. 53, 693 (2005).

13. S. Torquato, Annu. Rev. Mater. Res. 32, 77 (2002).

14. Y. Tian, T. Tan, Y. Wang, and Y. Fang, Pattern Recognit. 36, 649 (2003).

15. W. Dreyer, and W.H. Muller, Int. J. Solids Struct. 38, 1433 (2001).

16. Thermo-Calc Software AB, Thermo-Calc classic user's guide version $S$, 1 st edn. (Thermo-Calc Software AB, Stockholm, 2008), p. 1.

17. http://www.metallurgy.nist.gov/phase/solder/solder.html. Accessed 14 Jan. 2013.

18. S.B. Kim, and J. Yu, J. Electron. Mater. 39, 326 (2010).

19. M.W. Woodmansee, and R.W. Neu, Acta Mater. 54, 197 (2006).

20. P.S. Lee, H.R. Piehler, A.D. Rollett, and B.L. Adams, Metall. Mater. Trans. A 33A, 3709 (2002).

21. A. Tewari, A.M. Gokhale, J.E. Spowart, and D.B. Miracle, Acta Mater. 52, 307 (2004).

22. D.T. Fullwood, S.R. Niezgoda, B.L. Adams, and S.R. Kalidindi, Prog. Mater. Sci. 55, 477 (2010).

23. H.K.D.H. Bhadeshia, Stat. Anal. Data Min. 1, 296 (2009). 


\section{LIST OF FIGURES}

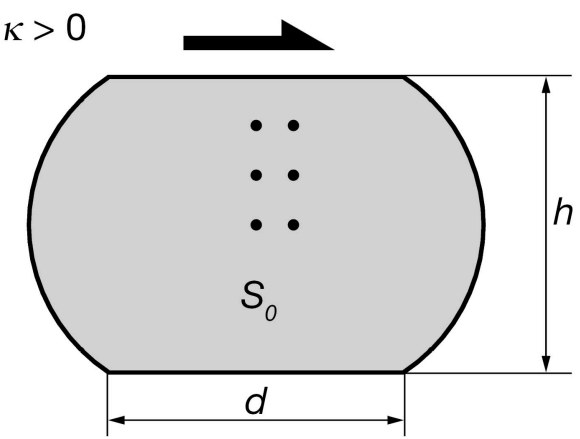

(a)

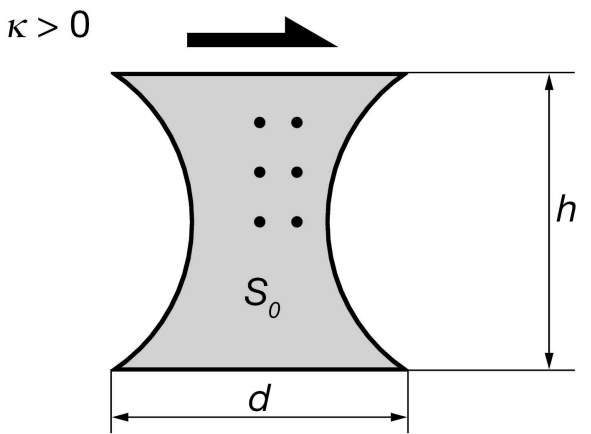

(b)

Fig. 1 The geometries of the solder joints studied in this work: (a) a barrel-shaped joint, (b) an hourglass-shaped joint. The arrows indicate the direction of the external shear load. The six dots in both (a) and (b) indicate the locations of the fluctuation of the mass fraction of $\mathrm{Sn}$ for the six different initial conditions.

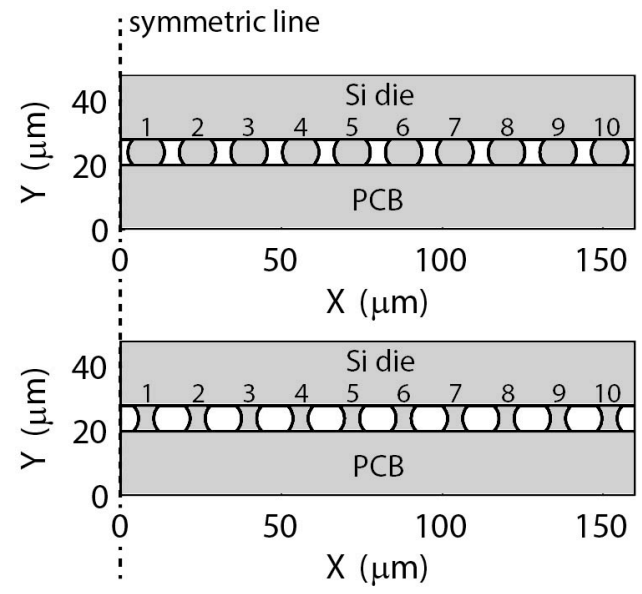

Fig. 2 Two packaging structures used for the simulations of thermal expansion.

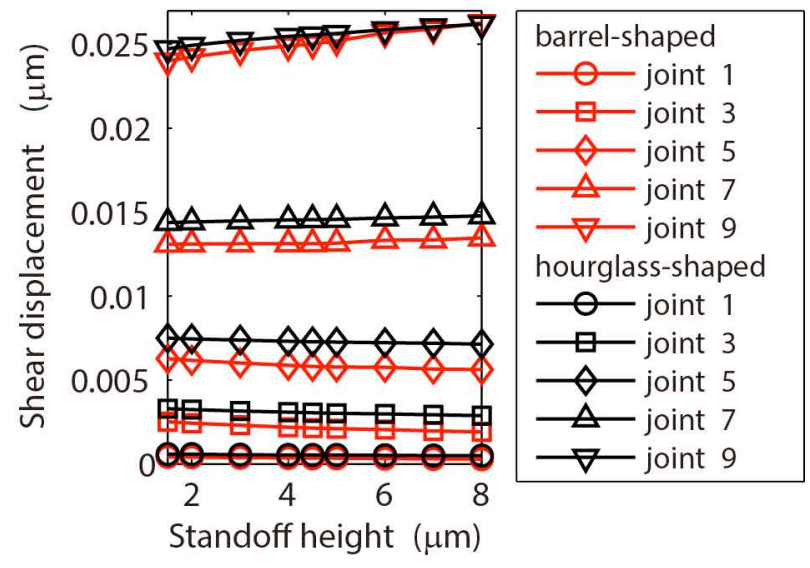

Fig. 3 The shear displacements of the top boundary of the solder joints shown in Fig. 2 . 


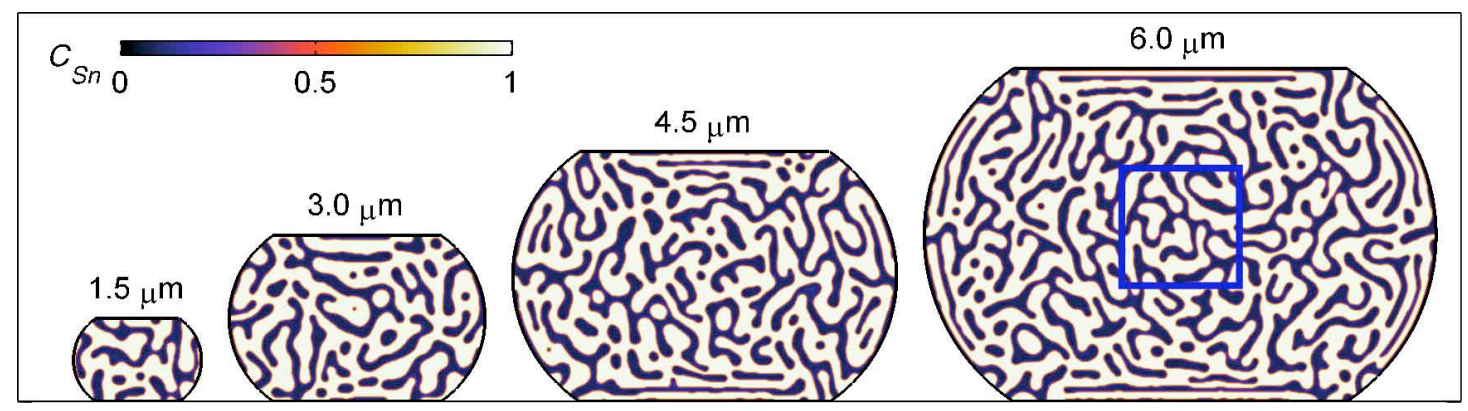

(a)

(b)

(c)

(d)

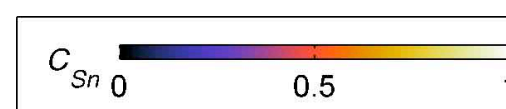

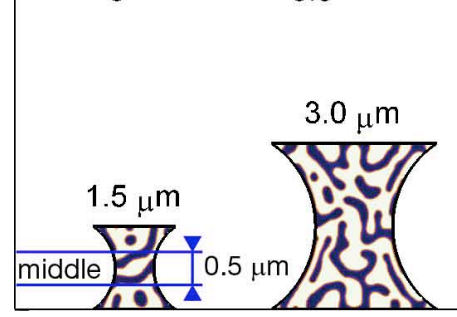

(e) (f)

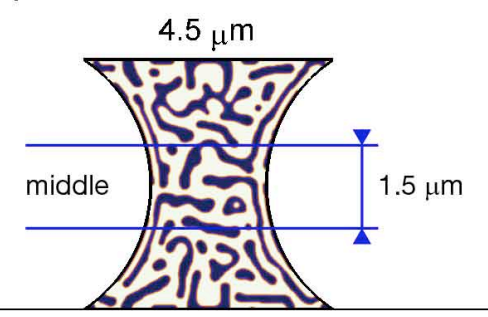

(g)

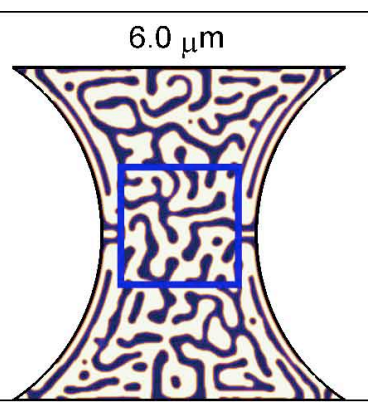

(h)

Fig. 4 The microstructures at $80 \mathrm{~s}$ during phase separation and coarsening at $125^{\circ} \mathrm{C}$ in (a-d) the barrel-shaped and (e-h) the hourglass-shaped $\mathrm{Sn} 37 \mathrm{~Pb}$ solder joints with different standoff heights. 


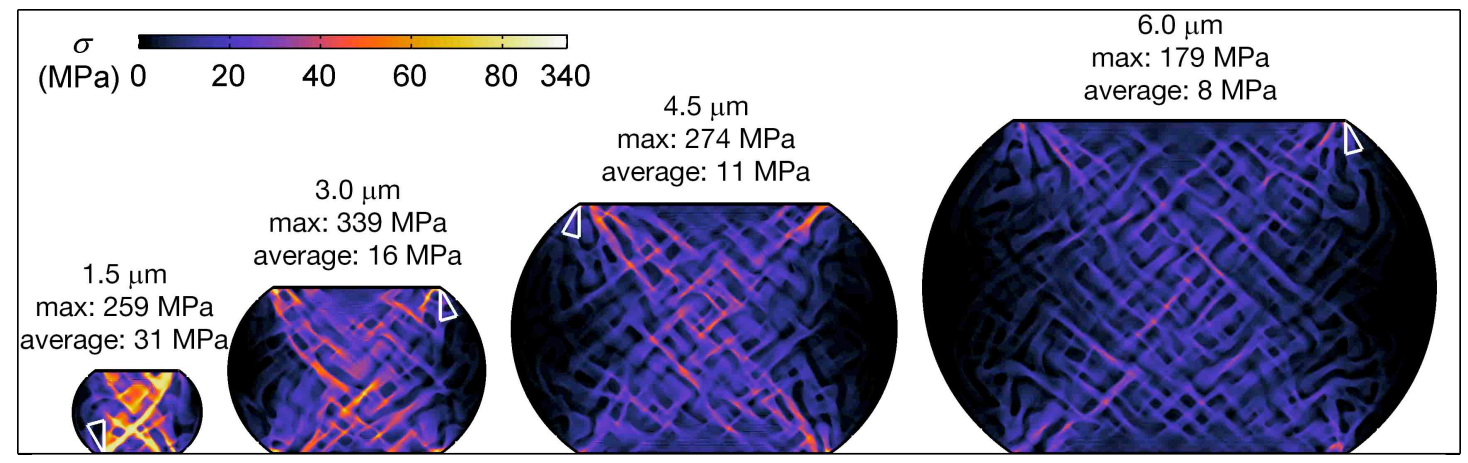

(a)

(b)

(c)

(d)

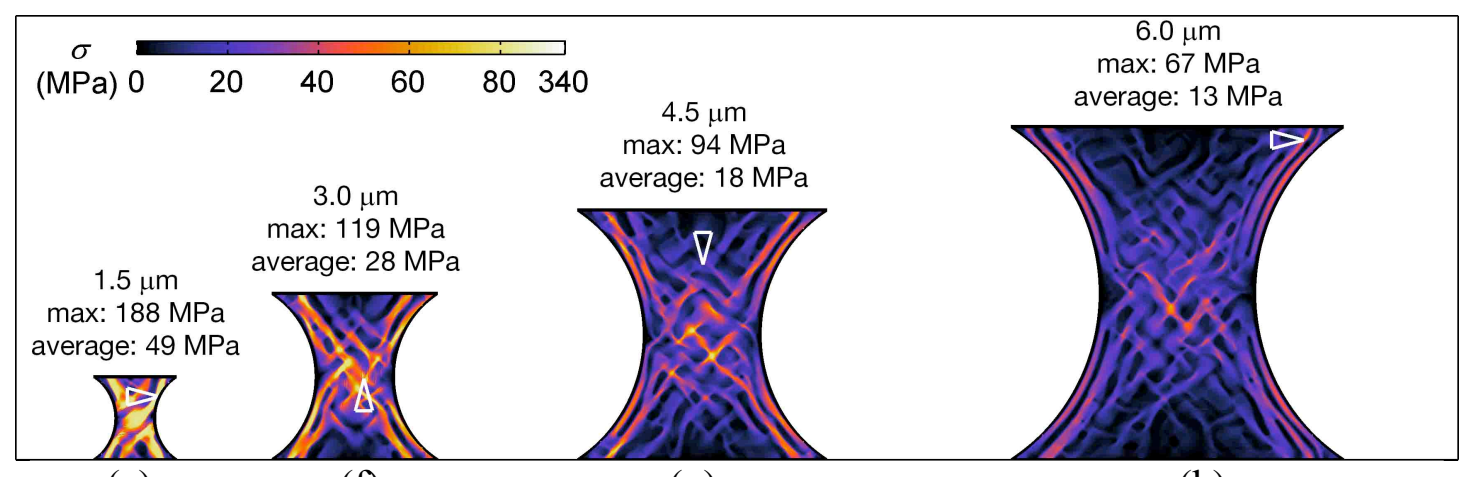

(e)

(f)

$(\mathrm{g})$

(h)

Fig. 5 The distributions of von Mises stress in (a-d) the barrel-shaped and (e-h) the hourglass-shaped solder joints of different standoff heights with the microstructures shown in Fig. 4. The values of the maximum and the average von Mises stress are shown on top of the joints. The locations of the maximum von Mises stress are indicated by the arrows. 


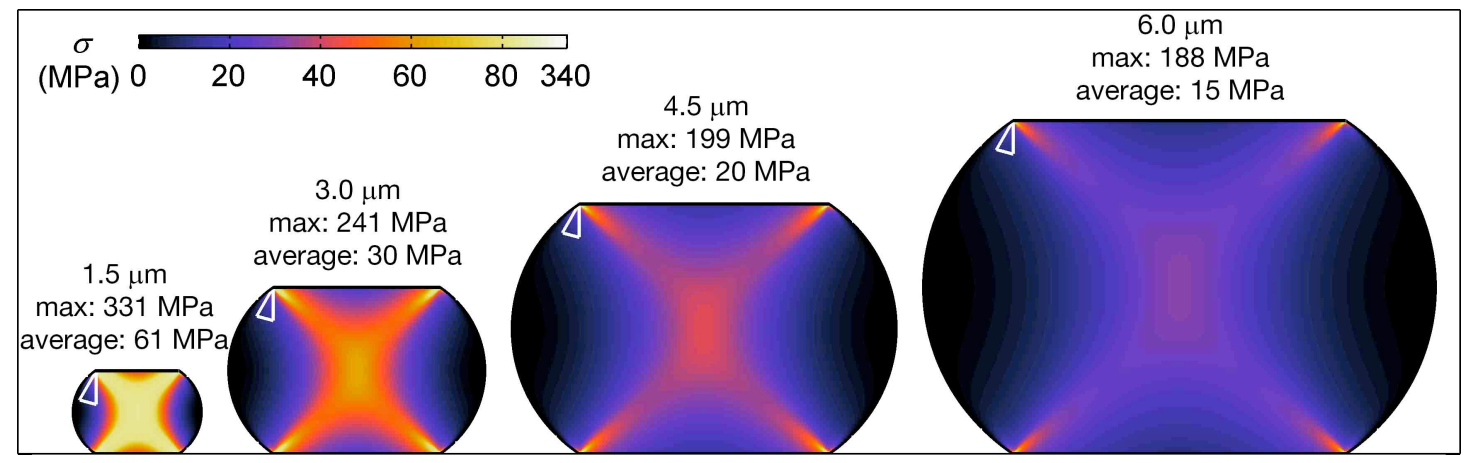

(a)

(b)

(c)

(d)

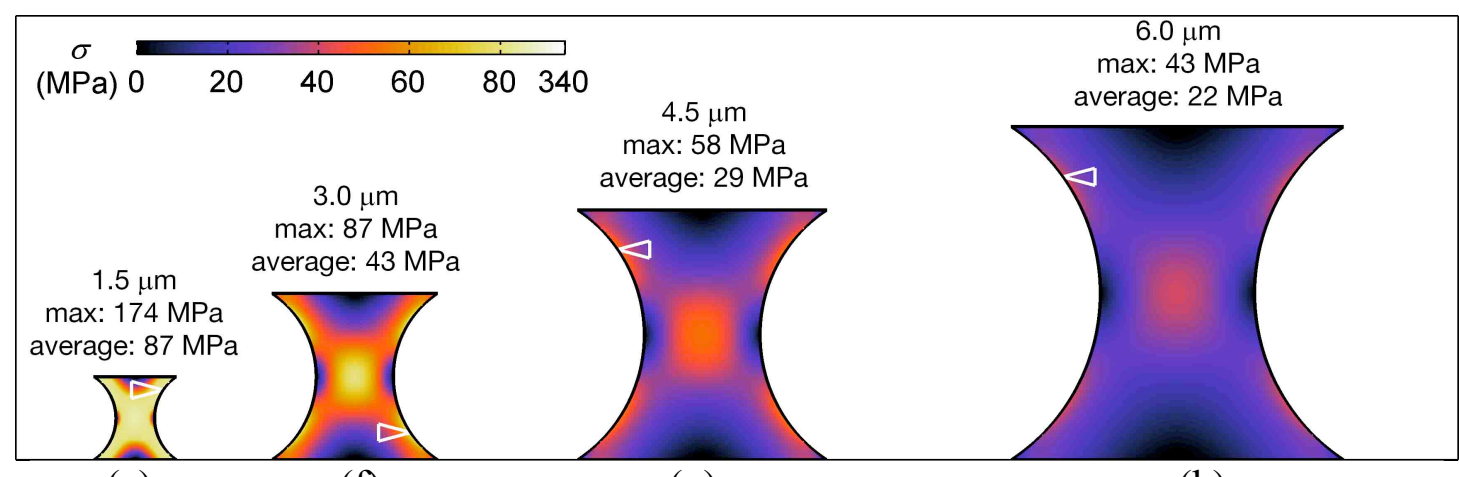

(e)

(f)

$(\mathrm{g})$

(h)

Fig. 6 The distributions of von Mises stress in (a-d) the barrel-shaped and (e-h) the hourglass-shaped solder joints of different standoff heights without microstructure. The values of the maximum and the average von Mises stress are shown on top of the joints. The locations of the maximum von Mises stress are indicated by the arrows. 


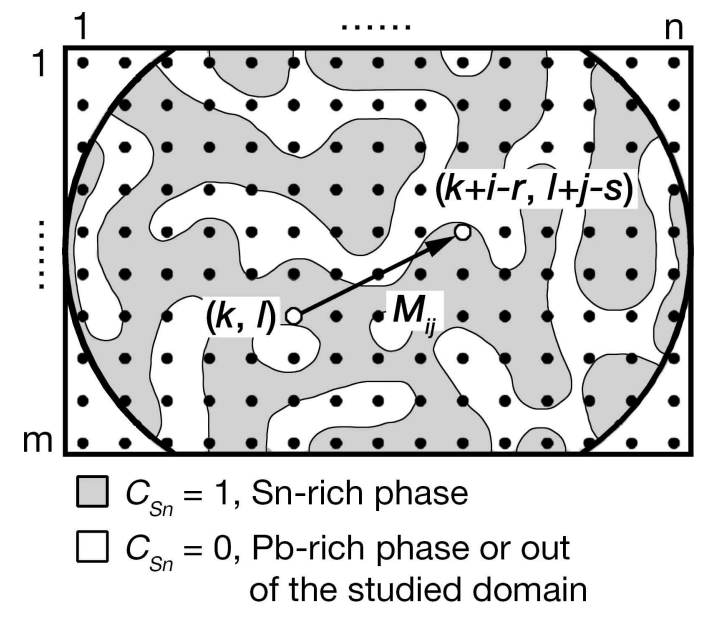

Fig. 7 A schematic diagram of a representative microstructural image studied in this work. The $m \times n$ pixels are illustrated as black dots. A vector $(i-r, j-s)$ is also plotted. The probability that the Sn-rich phase appears at both the start and the end points of this vector is described by the component $M_{i j}$ of the autocorrelation matrix. 


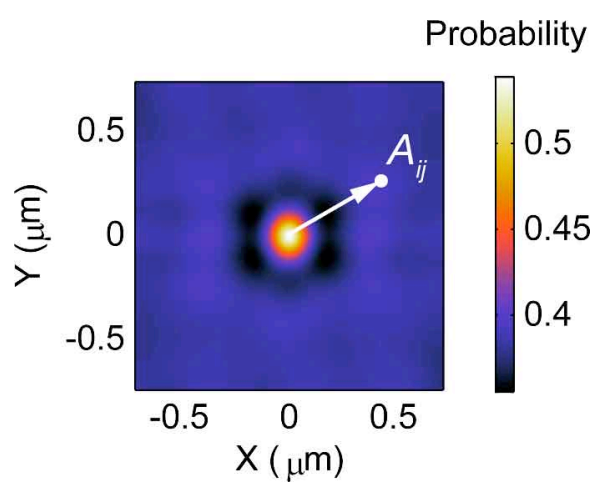

(a)

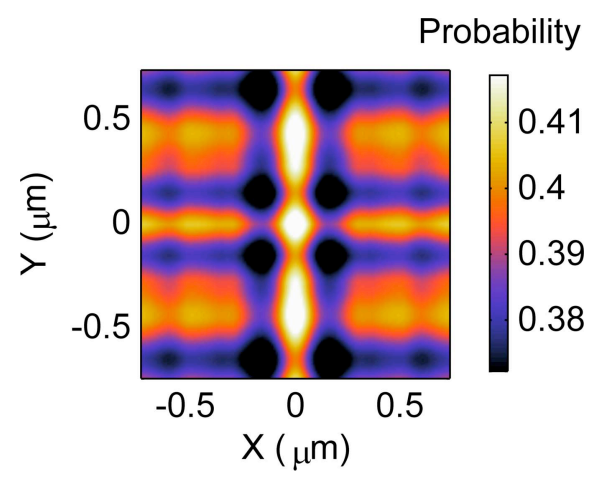

(c)

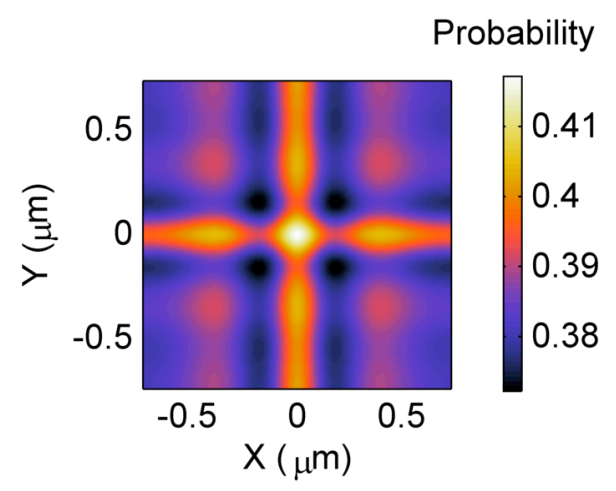

(b)

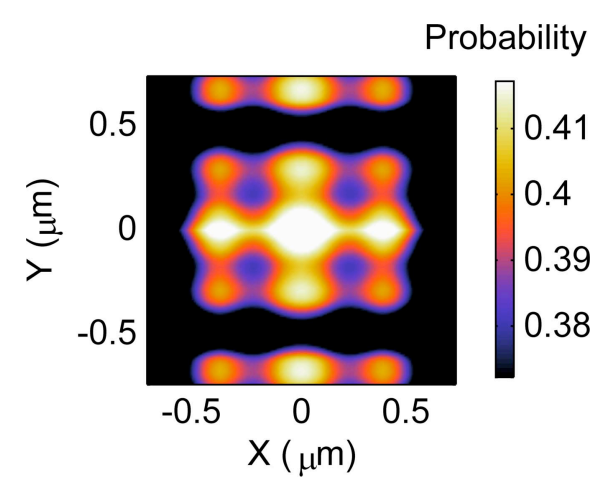

(d)

Fig. 8 The visualizations of (a) the benchmark $A$ of the autocorrelation matrices, (b) the first principal component of the benchmark, and the first principle components corresponding to (c) the barrel-shaped and (d) the hourglass-shaped joints shown in Fig. $4 \mathrm{a}$ and e, respectively. The value of the component $A_{i j}$ represents the probability of finding the Sn-rich phase at both the start and the end points of the vector shown in (a), when the vector is randomly placed in the microstructural image. 


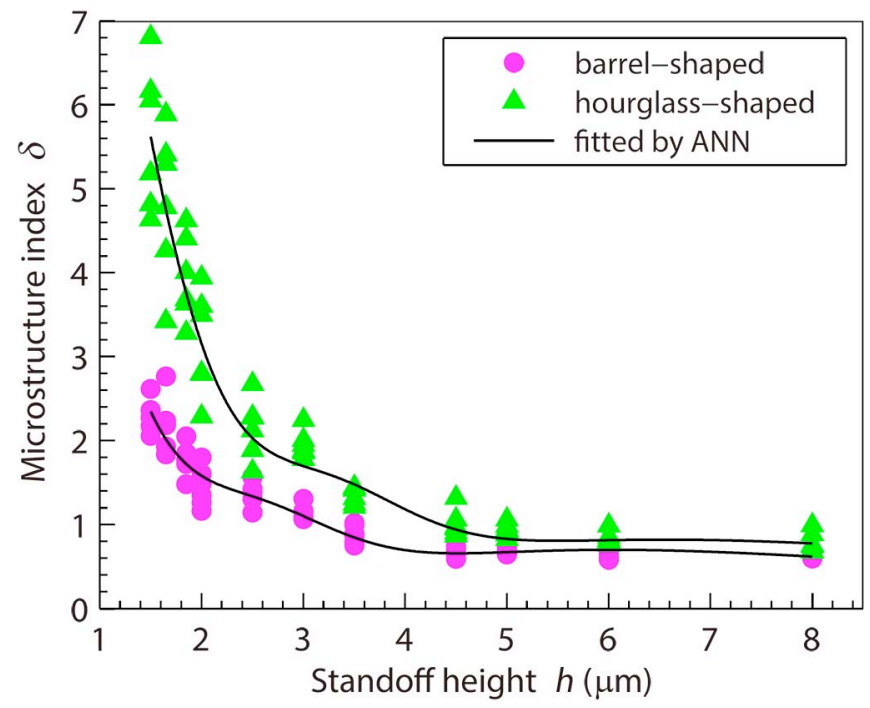

Fig. 9 The effects of the standoff height and geometry of the ultrafine interconnects on the microstructural index $\delta$. The microstructural index $\delta$ is calculated based on the simulation results that are plotted with solid circles and triangles, for the barrel-shaped and hourglass-shaped interconnects respectively. The relationship among the standoff height $h$, the shape factor $\kappa$, and the microstructural index $\delta$ fitted by ANN is illustrated as two solid lines. 


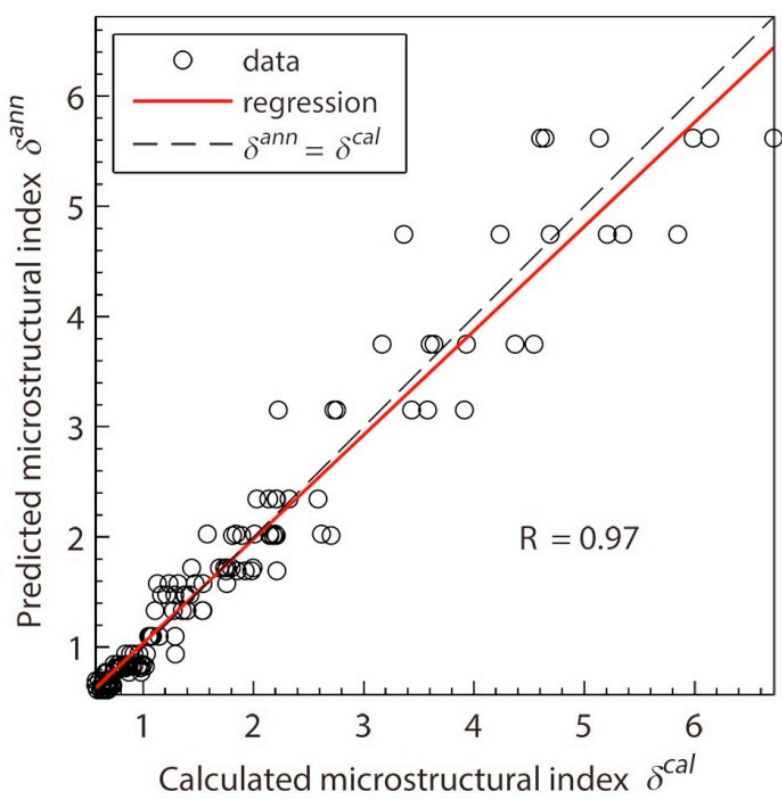

Fig. 10 A comparison of the microstructural indices predicted by ANN, i.e. $\delta^{a n n}$, and calculated based on the simulation results, i.e. $\delta^{\text {cal }}$. The data points, the line fitted by linear regression, and the correlation coefficient are illustrated. The dashed line represents the relationship that $\delta^{a n n}=\delta^{c a l}$. 


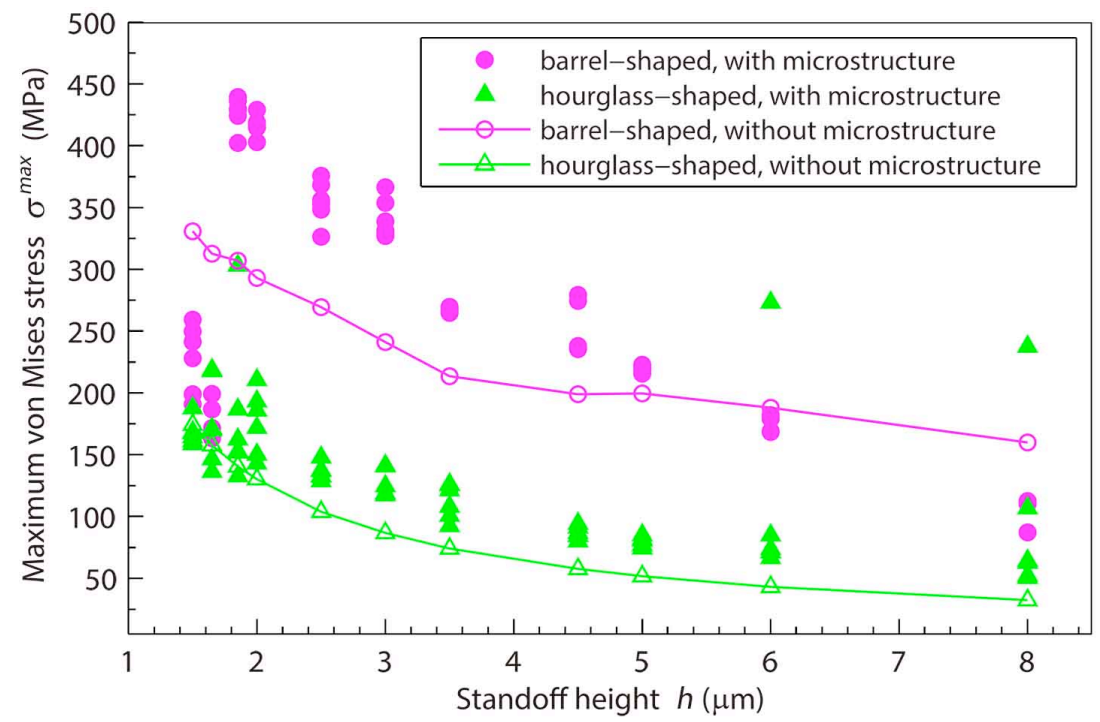

(a)

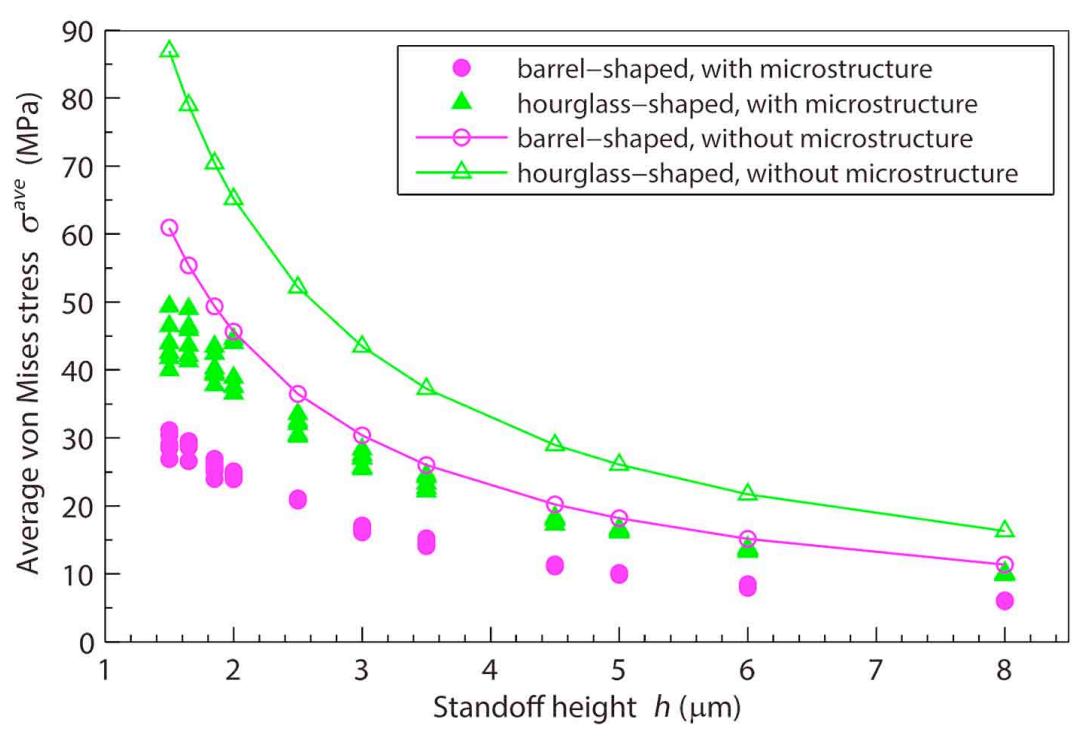

(b)

Fig. 11 The effects of the standoff height and geometry of the ultrafine interconnects on (a) the maximum and (b) the average von Mises stresses in ultrafine interconnects, i.e. $\sigma^{\max }$ and $\sigma^{a v e}$, respectively. The results for the interconnects with microstructure are plotted with solid symbols, and those without microstructure are illustrated with hollow symbols with solid lines. Circular symbols are used for the barrel-shaped interconnects, while triangular symbols are used for the hourglass-shaped ones. 


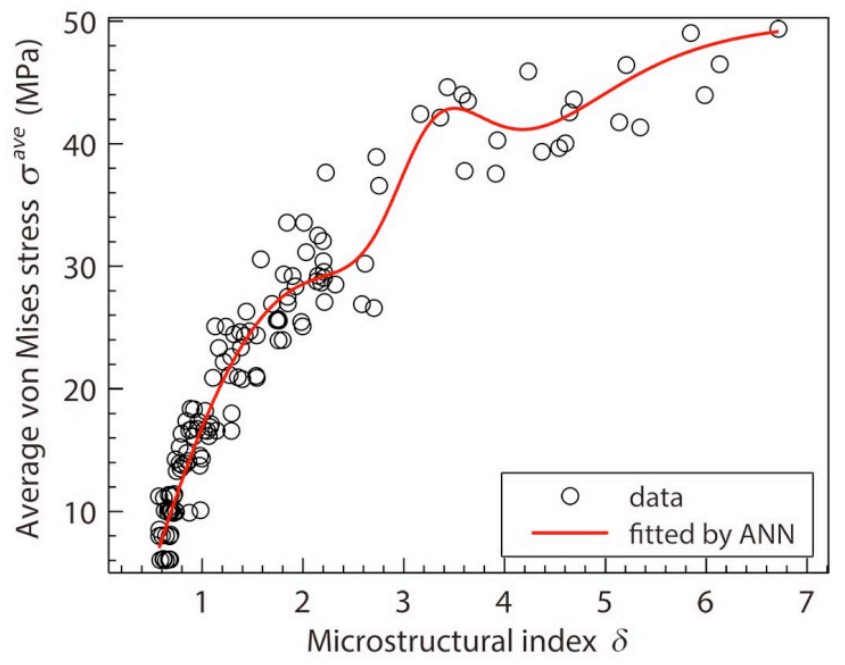

(a)

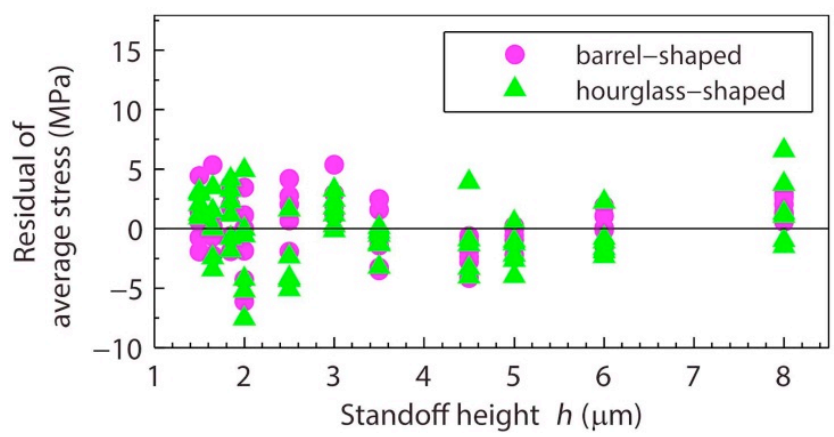

(b)

Fig. 12 A relationship between the average von Mises stress $\sigma^{\text {ave }}$ and the microstructural index $\delta$. The microstructural index $\delta$ calculated based on the simulation results is illustrated with circles, and the curve fitted by ANN is plotted with a solid line in (a). The residuals of $\sigma^{a v e}$ are plotted in (b) as a function of the standoff height $h$ for the barrel-shaped and the hourglass-shaped joints respectively. 